\title{
Empowering insight: The role of collaboration in the evolution of intelligence practice
}

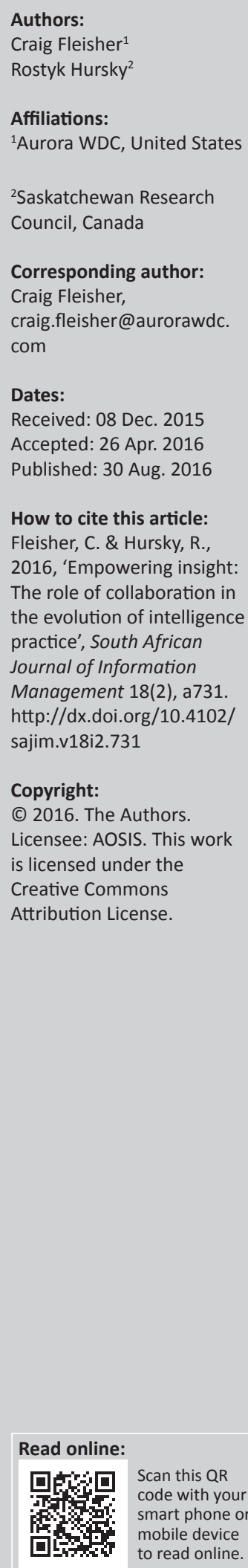

Background: Though subtle through the years, there has been a perceptible shift in competitive and market intelligence (CMI) practice from that of relying more heavily on sole operators to ones relying on collaboration. It happens within the nature of work performed inside intelligence functions, the larger organisation, and between organisations (i.e., intra-organisational). In this paper, the authors describe the change, develop a three-layered taxonomy for documenting it, and provide examples of how it impacts intelligence practice both now and possibly in the future.

Objective: To describe the increasingly evident role of collaboration and collaborative behaviour within insight producing functions in commercial, market-facing organisations. Identify evidence of collaborative intelligence practices in use across a range of different companies, industries, and geographies.

Method: The authors used a participant observation approach to developing this research. The discussion and frameworks in this study are based upon the authors' current roles, experiences and observations in leading a CMI group for a successful provincially based yet globally focused research and technology organisation, and having led interactive workshops and courses for over 100 organisations and approximately $1800 \mathrm{CMI}$ analysts in over a dozen countries.

Results: The authors identified an impressive array of collaborative practices for each of the three layers of organisational environments studied. These included ones in (1) intra-process (aka, intelligence cycle) collaboration, (2) intra-organisational collaboration (i.e. within the intelligence and broader organisation) and (3) inter-organisational collaboration (i.e. between discrete organisations). These are illustrated from actual, observed, and ongoing CMI practices and are shared as examples reinforcing our view of the movement away from independent practices and approaches toward purposeful, socialised ones.

Conclusion: The evidence we have amassed provides substantial evidence of a notable and beneficial shift from doing intelligence work independently, frequently within silos, towards doing it collaboratively and across multiple types of boundaries. Intelligence practitioners are growing in their capabilities by taking advantage of emerging technologies, adapting practices imported from adjacent fields and benefitting from academic and/or scholarly research that helps push ahead the working boundaries of the field and allows it to make progress. In our view, CMI practice has recently entered a third era of evolution, one in which collaboration will continue to feature prominently, if not centrally.

\section{Introduction}

\section{Problems requiring intelligence coordination and collaboration}

The days of the solitary or lone-wolf approach to intelligence have passed. Among the key reasons for this is that the context within which the practitioner works has shifted in ways that promote the need for collaborative effort. There are many challenges that occur as a result of the lack of collaboration among intelligence practitioners. Some of these challenges that have been prominent in our experiences are described in greater detail in the following paragraphs.

\section{Challenges caused by rivals that are increasingly complex, dynamic, fast-moving, boundary-spanning and sophisticated}

Contemporary rivals are typically not static or willing to utilise incremental tactics to compete over time. Many rivals are not going to be from geographic regions that used to constrain competition geographically, and this is multiplied by the ability of digital networks and exchanges to cut across physical boundaries. Today's rivals, especially ones that aim to compete on a global 
or multinational basis, are not as easily defined either, as many companies are an array of networks, alliances, partnerships, subsidiaries, public or private, contracts and/ or other arrangements that defy easy classification or typology. Some rivals are actually former, spun-off parts of one's own organisation! In this increasingly complex and fast-moving competitive context, it is critical that intelligence practitioners are able to collaborate across and span boundaries, and tap into factors and trends that were formerly beyond and outside the reach of any individual and their resources.

Because rivals and rivalry have gotten more complex and difficult to classify, many organisations have realised that their intelligence units do not have the resources to meet the competitive challenge being posed. Companies often have blind spots (Gilad 1994), face new competition, trends or rivals, and/or are not able to gather, analyse or communicate intelligence on a timely basis (Hackman 2011). This inflexibility in deploying practitioners or modifying competitive and market intelligence (CMI) applications and solutions, assuming these are available in the first place, has caused them to recognise collaboration as a desirable mode of operating practice.

Many intelligence problems and opportunities require a wide breath of perspectives (Heuer 2007). Collaboration is increasingly required when organisations face a context in which they lack a diversity of research or analytical approaches, lack employees who have a strong grasp of the craft of intelligence and have deep, practical experience, where only a few methods are consistently applied - thus limiting the range of insight or phenomena that can be adequately examined (Fleisher \& Bensoussan 2003), or where too limited thinking is routinely applied to problems (Hackman 2011).

In light of the steady flow of demands on their time and the ever-increasing flow of data they are required to process, individual practitioners can quickly get 'stale' in their approaches to their work (Fleisher \& Bensoussan 2003; Heuer 2007). Like any healthy field of practice, new developments are shared regularly by researchers, scholars and practitioners but absent a deliberate and formal approach to collaboration, the likelihood of a practitioner gaining awareness of this new knowledge is lowered. Additionally, lacking others to challenge and test their approaches, they lose the 'sharpness' of focus that is essential to effective intelligence processing (Hackman 2011). As such, intelligence practitioners have regular needs to continuously learn about new discoveries that can improve their practice and also to obtain focused training and development in order to just 'keep up' with developments in practice and context (Fleisher 2004).

Because of the exponential growth of digital data and information that has been generated in the last decades, many practitioners are no longer able to effectively filter, organise and analyse the inflows (Davenport, Harris \&
Morrison 2010). This so-called fire hose of information, and the sheer volume of increased noise further hides the soughtafter signal. Big data have overwhelmed many intelligence practitioners and units' ability to address it (Davenport et al. 2010). This phenomenon is an even bigger problem in companies that are customer facing, business to consumer focused and driven by the need to sell products and/or services. This has led to many of them seeking collaborations with, among others, professional service firms and specialised consultancies, taxonomical specialists, solution providers, aggregators and/or subscription vendors and other organisations to better respond.

Last but not least, many clients (i.e. the organisational executives or decision makers who seek out or request intelligence) have intelligence needs and problems that go beyond the ability of any sole intelligence practitioner to attain. Executives have the ability and frequently use their own networks or choose self-discovery processes they have conveniently available. For example, many executives are very comfortable using their own extensive human networks, going to a search engine, or accessing increasingly sophisticated dashboards and reporting portals. Many of these efforts are deemed to be insufficient; thus, they refer these matters to their intelligence staff. The staff member must then fully understand what actions were used by the executive and then extend their reach beyond those in order to generate higher insight quality. This can rarely, if ever, be done in the absence of collaboration.

\section{Key benefits gained from intelligence collaboration}

Our observations and experience have demonstrated that there are numerous benefits that come out of collaborative behaviour, whether among individuals inside the organisation or between different organisations in the larger commercial marketplace. Among those that witnessed most often include the following 10, listed in no specific order:

- Collaboration ties people together for the greater good: Collaboration enables entities to tackle problems that are too big for any single entity operating independently.

- Financial benefits: Collaboration can let a CMI unit stretch its resources. A good example of this is when intelligence engages individuals from outside its fulltime roster, thus enabling intelligence gathering to include a wider base, more networks and extend far beyond the aegis of the full-time team members.

- Subject matter benefits: Collaboration has enabled CI employees and their units to gain access to otherwise unavailable topical domains and stretches the knowledge basis of existing and/or permanent employees.

- Team-building benefits: Collaboration can strengthen individuals' skills, as well as 'stretch' practitioners to develop competences that would go beyond what they might otherwise achieve.

- Time benefits: Collaboration can give an intelligence unit the ability to produce intelligence more quickly, particularly when those collaborations cross time zones. We know of some organisations that keep their CMI 
projects active and working 24 hours a day because they have their Asian unit work it for 8 hours, their European unit takes over the next 8 hours, and their American unit follows through the next 8 hours, until it gets returned back to the Asian unit to begin the process anew the next day.

- Bias reduction: Collaboration with a greater number of arms-length or neutral participants can provide a CMI team with the ability to remain neutral and deliver a perspective on the intelligence in an environment that can become driven by personal interests, growth drivers and 'pet projects'.

- Linkages with other organisational processes: Reliable and effective insights that can be directly linked to the Enterprise Risk Management or Collaboration Systems, Customer Relationship Management Systems and other Management Information Systems.

- Analysis breakthroughs: Collaboration enables the development of new analytical models, techniques and approaches, some of which cannot be effectively done by individuals, if at all.

- New perspectives: Collaboration has provided intelligence teams with the ability to see a perspective from other stakeholders' eyes, utilise lessons learned and come up with best-case scenarios to adapt or apply to one's own organisation.

- Validity and generalisability checks: Collaborations with individuals from various sectors, industries and professions provide intelligence and insights on industry convergences or divergences.

\section{Types of collaboration within intelligence practice: Intraprocess}

There are a variety of different forms of collaboration used by intelligence practitioners and units (Hackman 2011). These range along a continuum of the degree of collaborative intensity. In other words, some forms are less intense, suggesting more informal means of regular communication and interaction, as well as a lesser degree of formalised organisational or bureaucratic mechanisms established in support of it. Others are far more intense, characterised by a high degree of formalisation, the use of scheduling and project management methods, supported by change management practices and designed to last for longer, if not indefinite, time periods. These different forms are illustrated in Box 1 (see Box 1).

We have witnessed CMI units making use of all these collaborative forms. Arguably among the most utilised of these would be Communities of Practice, as organisations create subject matter expert (SME) networks of individuals,

BOX 1: Continuum of intra-organisational collaboration of intelligence functions.

Less Intensive Collaboration: $\rightarrow$ Community of Shared Interests (e.g. both internal and external subject matter expert networks), Communities of Practice (CoPs - such as R\&D practitioners, regulatory advisors, sales personnel, etc.), Emergent Collaborations, Coaching Groups, Distributed Teams, (Special/Designated) Project Teams/Task Forces, Semi-Permanent Work Teams. $\rightarrow$ More Intensive Collaboration. Source: Adapted from Hackman, J.R., 2011, Collaborative intelligence: Using teams to solve hard problems, Berrett-Koehler Publishers, San Francisco both inside and outside the organisation, that help intelligence staff members track, monitor and assess emerging developments in specific practice areas. These networks perform both regular/routine as well as focused reconnaissance and can be made quickly available for special projects or requests. Another increasingly used form in intelligence practice is the semi-permanent insight team as described in case detail by Fleisher, Wright and Allard (2008). These are semi-permanent work teams that cut across organisational boundaries and are designed to bring crossfunctional, cross-geographic perspectives to planning and strategy development.

Certain forms of collaboration have demonstrated to be especially promising in intelligence applications, those being multidisciplinary and interdisciplinary forms. Multidisciplinary collaboration means working with several disciplines but with individual goals and where the different disciplines work independently. It does not challenge disciplinary boundaries and the participants learn about each other (Choi \& Pak 2006). Interdisciplinary collaboration occurs when others' boundaries are being blurred and the participants learn about and from each other (Choi \& Pak 2006).

There is also a changing nature of collaboration applied with the intelligence cycle (Hackman 2011). The analysis process is a pertinent example of how this changed the nature of insights delivered by practitioners. Past competitive intelligence analysis practice emphasised independent, individual analysts producing insights. Today's analysis is more frequently generated by groups, in shared work sessions, whether in-person and face-to-face as led by facilitators or done in on-line platforms that allow for synchronous analysis to take place.

Even the nature of analytical methods used has been changed by collaboration. More social analytical methods such as war gaming, scenarios, blue and/or red teams and shadowing get greater emphasis in today's collaborative intelligence process. Even older, more static methods like SWOT, four corners or competitor profiles benefit from active collaboration by SMEs in networks using a variety of digital communication channels and specialised software applications to enliven the outputs and keep them evolving as developments warrant (Fleisher \& Bensoussan 2015).

\section{Intra-organisational collaboration}

When business, competitive, economic and/or market intelligence first came to prominence as an outgrowth of public intelligence practices applied in military and defence endeavours, it was often practiced by the sole or 'lone ranger' analyst, data collectors and often managers who worked on their own and had to exhibit a wide range of different skills to execute the entire intelligence cycle. Early comprehensive research surveys of CMI practice conducted under the auspices of Strategic and Competitive Intelligence Professionals (SCIP) and related international intelligence organisations demonstrated that many earlier era business, 
commercial or corporate units consisted of one individual who 'did it all' (Prescott \& Fleisher 1991) - and even these surveys greatly underestimated the number or percentage of sole operators because of sampling issues (i.e. surveyed SCIP members, who were more likely than the population member to work in a larger business or corporation).

Intelligence units of one were frequently tasked with developing the mission, meeting all their client's needs, developing the essential intelligence resources such as the databases, networks, subscriptions, systems and the like required to execute the basic CMI process (West 2001). Analysts were sometimes viewed as the 'geniuses' in cubicles or behind their desks, crunching the data and numbers until the wee hours of the night, working with mysterious formula, and doing 'black magic' with their 'black boxes' to produce their insights. Although we still see intelligence units-of-one show up in empirical studies of practice, hardly any CMI practitioners can be effective without working with others inside, and outside, their organisations. Today's intelligence reality shows practitioners frequently working collaboratively in teams, whether doing networking, source identification and validation, data fusion, training, special projects, management, sophisticated analysis methods like red and blue team exercises or war gaming, scientific and technology scouting or just developing enhanced insight deliverables (Fleisher et al. 2008; Hackman 2002, 2011).

The movement towards intelligence collaboration within and among intelligence teams began to happen in the late 1980s and has accelerated in its evolution since that time. Modifying an approach first described by Fleisher and Bensoussan (2015:62), we suggest that there have been three distinctive eras that the organisational aggregation of expertise has taken.

The first era of business and commercial competitive intelligence was nearly always done by individuals working in silos. These practitioners were expected to work intelligence problems through the entire intelligence cycle of planning, data gathering, analysis and dissemination. Collaboration, to the extent that it occurred, was nearly always practiced in a serial or sequential manner. Intelligence was generated based more on a scale basis than a scopebased one. Simpler intelligence problems, or ones that existed within mostly mature and stable contexts, and where there was a lesser degree of VUCA (aka, volatility, uncertainty, complexity, ambiguity) could be reasonably addressed through these processes. This generation was highly paper intensive and lasted until the early 1990s.

The second era of collaboration occurred following the rapid individual and organisational adoption of information technology (IT) resources and particularly those used for data transmission and information communication. E-mail, the Internet and World Wide Web, faster processing power, less expensive memory, connected desktop and organisational networks and portals, allowed for more synchronous communication around the organisational management and processing of intelligence matters. These enabled more collaboration to occur in a timelier and more frequent fashion and permitted some intelligence units to begin gaining scope advantages in their gathering (especially through automated agents or 'push' means) and processing of intelligence. Collaboration still had not achieved full and continuous levels of interactivity (e.g. $365 \times 24 \times 7$ interaction), and the IT being utilised continued to improve at uneven rates, but intelligence executives had not yet captured the full potential benefits of collaboration throughout the intelligence cycle. This generation was dominant from the early 1990s until around the middle of the first $\mathrm{Y} 2 \mathrm{k}$ decade.

A third distinctive era of intelligence has occurred since the latter part of the first $\mathrm{Y} 2 \mathrm{k}$ decade. It arose concurrently with the rapid commercial growth and widespread adoption of mobile communication technology, big data technologies and enterprise collaboration systems (ECS). Smartphones, faster networks, social media (SM), transaction using mobile devices and the bigger, more detailed, timelier data it generates have been enormously helpful to intelligence practitioners. Adding to these cloud storage and access, geolocational data developments, more powerful processing and a resurgence of artificial intelligence, machine learning, data science methods and the conditions are fertile for a highly collaborative form of intelligence operation. This generation is in evidence at some of the more advanced intelligence operations, though it is still not yet predominant among most business and commercial CMI groups.

ECS used for intelligence collaboration purposes would include combinations of groupware, software tools, the Internet, extranets and other networks needed to support enterprise-wide communications. ECS allow for the sharing of documents and knowledge to specific teams and individuals within the enterprise and are a key factor in third-era CMI practice. Enterprise collaboration software applications enable intelligence team members to collaborate on an ongoing basis by allowing them to share and/or coordinate updates to documents any time regardless of where the members are located. Examples of collaboration software include e-mail, enterprise blogs and wikis, instant messaging (IM), online whiteboards, videoconferencing, collaborative document sharing and project management tools.

Third-era intelligence collaboration functionality tends to include choices from among the following five categories:

- Messaging: E-mail, calendaring and IM.

- Team Collaboration: Intelligence portals, document repository, project management, library services, workflow and discussion threads.

- Web Conferencing: Virtual meetings, video and audio teleconferencing, shared desktop and presentation.

- Shared Data and Analytics: Cloud storage, collaborative analysis, automated filtering.

- SM: Blogs, social networking, wikis. 
Multinational companies, such as Microsoft, Cisco, Web-Ex, Citrix, IBM, SAP, Oracle and others, are actively improving existing applications and developing new programmes designed to enhance collaborative productivity in this space. Additionally, these companies' solutions have also been augmented by Voice-Over-IP offerings, such as Skype, and SM offerings, such as Facebook, Instagram, Twitter, blogs and wikis. These have nearly unlimited potential to drive forward third-era intelligence collaboration.

New IT and software solutions also allow for individuals to build upon others' work while they are asleep, to autonomously update reference sources, to fuse knowledge across different data types (e.g. visual, signals, text, voice), to tap into previously inaccessible networks, to filter and move the data and information around seamlessly and instantaneously and to engage in and apply synchronous analysis methods.

For intelligence units, embracing collaboration technology is at the heart of managing data, information, intelligence and knowledge. The organisation benefits by unlocking, synthesising and sharing knowledge hidden among its employees and also discovering new sources of expertise. It also plays a fundamental role in bringing together the workforce, whether local or global, by enabling the creation of both social and professional communities of interest, and across traditional functional, geographic and market boundaries.

Linking collaboration to business processes and high-level business objectives gives intelligence staff valuable insight into the larger enterprise business scenario. This supports more effective, efficient and timely decision making. Though we are still in the earlier stages of this generation, the promise for IT, solutions, SM, VoIP and increasing mobility in intelligence remains very large and agile, early adopters stand to gain at least temporary, short-term advantages in applying the third-era resources to boost the collaborative learning potential of their intelligence processes.

\section{Collaboration as part of the generation of organisational learning or wisdom}

Collaborative learning cultures serve as a foundation of effective executive leadership through encouraging reflection and learning at all levels of the organisation (see Figure 1). Work cultures are generally viewed to be the atmosphere or climate of a work environment, perception of how it feels to work in the organisation, within a particular team or for a specific supervisor and/or the ideal operating environment required to provide a sustainable, highly effective work climate that optimises the team's potential to do its best work (Wilderom et al. 2011). Unfavourable work cultures tend to generate frustration, which is projected onto others, resulting in tense and problematic relations and contributing to poorer outcomes for decision makers and executives.

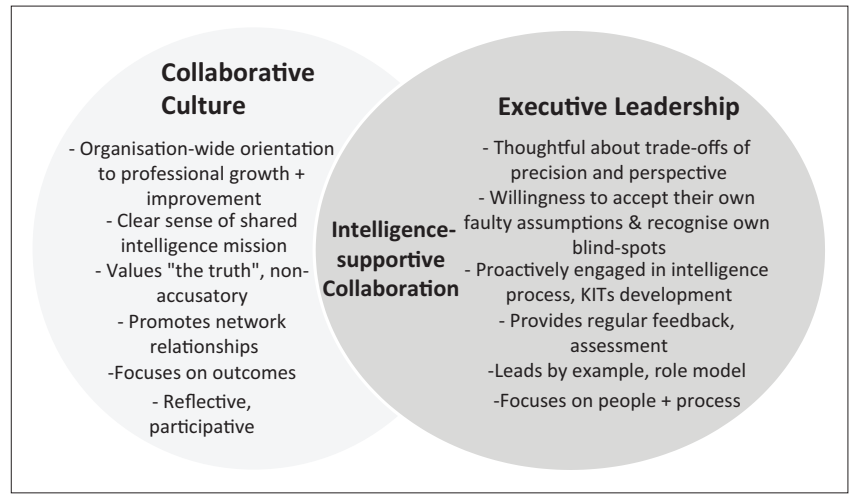

FIGURE 1: Collaboration as part of an intelligence-conducive work culture.

Many individuals' fundamental organisational needs revolve around clarity, development, meaning, purpose, development and connection. After extensive research and analysis, Gallup identified critical factors that contribute to practitioners' productive motivations, engagement and outcomes (Cotton 2012; Cotton \& Hart 2011; Hart \& Cotton 2003; Wagner \& Harter 2006). These factors refer to practitioners:

- having clear expectations about their roles and responsibilities

- having the basic material resources to undertake work

- having regular opportunities to utilise their talents, strengths and aptitudes

- receiving feedback and recognition for good work and progress

- having a supervisor who shows interest in their professional and personal development

- having a say and having their opinions taken into account

- feeling that their role is important to the organisation

- having a commitment to the work group in order to undertake quality work, and

- having a sense of connection and someone in the organisation to confide in.

Teams can provide benefits for both organisations and collaborating employees through higher productivity, quality improvements, greater flexibility and speed, a flatter management structure, increased employee involvement and satisfaction, and lower turnover. However, teams often present greater leadership challenges than does a traditional hierarchical organisation.

\section{Summary of intra-organisational collaboration}

Reflecting on our observations and experience, we have identified several necessary conditions that organisations should have in place to be effective in this team-supportive, collaborative role.

\section{Having the right teams in the right places}

In his book Leading Teams, Hackman (2002) makes a distinction between 'real teams' and 'co-acting groups'. Applying his definition of co-acting groups to business organisations, practitioners may have offices located next to one another and regularly meet to share ideas, but each practitioner's analyses and deliverables are independent of the others. 
However, in productive teams, members share the responsibility for the success of their decision makers. Team members rely on each other for increasing the abilities of all organisational clients. They do not view their roles as being 'my clients' and 'your clients' but rather see it as being 'our clients'. In intelligence functions, interdependence for business outcomes is the most critical hallmark of collaboration.

'Real' team members tend to talk constantly about what they have in common. Today's better intelligence solutions include collaborative communication technologies such as real-time chats, IM and real-time video teleconferences that allow for this constant communication to securely occur between organisational members. Because similarly experienced or tasked analysts, managers or researchers share regularly about their work processes, needs, networks, standards and assessments, interactive, collaborative communication mechanisms are usually the most productive configuration for effective intelligence analysis.

\section{Building a culture where trust is central}

A significant level of trust must exist among team members and between the C-suite and other executives. Executives need to empower their intelligence staff to act decisively to raise new and powerful insights, as long as their recommendations and insights can be supported by data. As Richard DuFour pointed out in Learning by Doing, even though this level of trust takes time to generate, leaders cannot wait until the perfect positive culture is in place to have intelligence operatives start the data dialogue. Struggling together to initiate the conversation - using both face-to-face and digitally supported means, implementing the decisions made during the conversations and dialogue and, most importantly, seeing executive success and support increase, are effective builders of trust.

\section{The use of protocols}

Having an agreed-upon process in place can be useful to structure difficult group conversations. In his publication The Power of Protocols (McDonald et al. 2013), Joseph McDonald and others define protocols as 'guidelines [that] everyone understands and has agreed to, leading to conversations that school people are usually not in the habit of having'.

Using this protocol, intelligence teams answer a series of questions that guide their analysis of business problems, opportunities and outcomes to identify (1) patterns of organisational intelligence strengths and weaknesses in colleagues' understanding of the deliverables being generated; (2) individual intelligence practitioners ready for enrichments and interventions, and the professional development focus that the differentiations should take; and (3) practice improvements and upgrades they will make. A focused exploration of powerful questions in a logical sequence enables intelligence teams to begin to develop the skills they need to be collaborative.

\section{Articulate compelling reasons to perform collaborative analysis}

As leaders in academe and practitioner organisations, we have pressed staff and our colleagues to conduct data-driven conversations. But in some cases, we may not have provided what Hackman (2002) and others labelled a 'compelling direction' to energise team members and engage their talents.

It is becoming increasingly clear that justifying data analysis to intelligence practitioners based on increasing satisfaction and meeting adequate unit progress or arbitrary goals is far less persuasive than basing improvement initiatives on the reason most intelligence practitioners remained in the profession - to help executives make better decisions and to underlie successful organisational actions that generate new benefits, prosperity and wealth.

\section{Clarity about team autonomy}

To enable collaborative teams to be successful, leaders must be clear about the extent of autonomy team members will have to act on the results of their dialogues, such as challenging essential assumption, concepts, practices or altering the nature of future deliverables. Either CI practitioners have the autonomy to make insights based on their data as it is processed or they must follow pacing guides that identify what must be regularly delivered. They cannot do both at the same time.

It must be clear to intelligence employees that insight teams are expected, not just permitted, to spend additional time on significant opportunities or problems that arise and/or they have identified, that some executives may not have observed or properly digested, and to modify the content, timing, intelligence methods and/or future deliverables or project assignments, based on documented client needs.

\section{Provide ongoing coaching, mentoring and support}

Because working in networks and teams can be chaotic and challenging, regular coaching from leadership team members and mentoring from more seasoned teammates is essential to sustain top performance. Process feedback about the internal operation of teams is particularly important as teams begin work in the early stages of their collaboration and struggles to establish a more collaborative environment.

\section{Integrate self-accountability into their ongoing operations}

Most teams require assistance in putting internal mechanisms in place to follow-up on outcomes decided upon during interactions and dialogues. Effective protocols often include templates that teams complete as their conversation progresses - a recommended part of the Key Intelligence Topic and/or Key Intelligence Question development process for planning intelligence projects (Herring 1999) as well as reference interviewing such as those done by special librarians (Ross 2003). As meetings end, the chair e-mails to the members the template containing the actions team 
members are committed to take. A copy can be forwarded to executive clients so they can better monitor and support the team's work and also allow for collaboration and discussions as the intelligence employees work through their CI process.

Follow-up is improved when team leaders document at the next team meeting the outcomes of the process improvements that they implemented. Assessment activities such as shared work sessions, 360-degree feedback, deliverable debriefs, walk-throughs and periodic reviews by the team with their executive clients can also serve to increase internal team accountability for follow-through (Rao 2014).

\section{Communicating successes, regularly}

Most business professionals recognise the importance of celebrating achievements, even the so-called smaller ones, during the early stages of any change process. However, an essential part of celebrations often overlooked is the intentional connection by executives of the specific actions of intelligence staff, such as increased collaboration across geographic and functional boundaries and leveraging structured data analyses, to the business outcomes and results that followed.

Where might an organisation identify its most potent leverage point be in increasing intelligence team performance? When a CI director reflects on the effectiveness of CI teams, it is worthwhile to determine whether their organisation's executives can answer the following queries:

- Do the intelligence teams act on a clearly articulated, compelling direction to reduce performance gaps by analysing data collaboratively?

- Are conversations, KIT development, reference interviewing and other data dialogues structured by protocols that lead decision makers to take definitive customer-focused, market-impactful actions that increase revenues and profitability?

- Also, are intelligence team members clear about leaders' expectations that they act decisively, as the data warrant, to modify their instruction to address identified weaknesses?

- Do team members have in place their own internal accountability mechanisms that result in follow-up and thoughtful reflection on what works and why?

- If these questions have definitive answers, it is a helpful indication that they will achieve higher levels of executive satisfaction and impact than those organisations that struggle with answering them.

\section{Inter-organisational collaboration}

The data and information underlying intelligence are generated every time individuals and/or organisations make a business or commercial exchange. More of it is created on a daily basis than in digital formats and around the world, then at any time in human history. In today's increasingly globally competitive marketplaces, no organisation can operate for long in isolation. Inter-organisational networks are increasingly used to accomplish business and commercial tasks, whether these are used for financial exchange and transfer, information transmission, logistics and in allowing the movement of human resources and skills. Intelligence practitioners and insight teams must increasingly work across differing cultures and languages, national and regional markets, regulatory frameworks, trade policies and the like. Very few organisations have the global expanse of resources to gather, process and analyse intelligence across all of these boundaries; therefore, collaboration has become an essential and fundamental element of conducting effective intelligence processes.

Managing networks of organisations has become a fundamental task for managers who seek to maximise their own organisation's intelligence potential. In the earlier years of the CI field, organisations used to try to manage and own as many of their own resources as possible in conducting intelligence operations. Whether this was hiring their own CMI practitioners, having an internal library or knowledge centre, maintaining large assets of subscriptions to informational resources and databases, the ability to have an independent CMI operation within larger commercial enterprises was a point of organisational pride and sometimes even thought to confer business or commercial advantage. If resources were needed to address intelligence challenges that went beyond the reach of the organisation's own resources, strict agreements could be drafted to allow for the temporary hiring of professional services firms, consultancies or other advisory bureaus. These often satisfied the needs, but frequently created a new set of transaction costs or agency problems that required new and often innovative managerial responses (Wood \& Gray 1991).

In today's data-driven, insight-seeking, increasingly competitive business world, the prevailing approach to intelligence efforts, especially from organisations that do not have the 'we've got unlimited money and resources to do it all in-house' mindset, has been to seek out experts, networks and linkages to individuals who have and are willing to share insights, opinions and perspectives relative to a specific challenge or a question (Rowley \& Gibbs 2008).

It can be argued that the establishment of collaborative intelligence networks and expanding CMI efforts outside of one's own organisation has been reliant solely on the CMI head within the organisation. These individuals have the awareness and whereabouts to be able to strategically develop a compelling business case for each collaborative effort and how they would benefit both or multiple stakeholders. The authors believe that the current external environment is generally not yet built and predisposed for naturally occurring collaboration with intelligence initiatives. Someone has to initiate and persistently move the effort forward, being well aware that some collaborations will come naturally, while others will take time to develop. Even when collaborating makes complete sense and the organisation does not know how it ever made it without such an effort, 
the natural tendency is still to try and do it alone or wait for someone to instigate the discussion.

In most instances, the CMI practitioner is really well positioned to instigate thoughts and discussions about collaboration, both within and outside of the organisation. They should be well aware of some if not most of the major stakeholders locally and potentially globally who can collaborate with them on some intelligence efforts. For smaller organisations, global CMI networks provide access to thought leadership, guidance and advice on how to approach collaboration. They also provide access to direct contacts one would never be able to connect with otherwise. Local networks require more knowledge building and educating about CMI efforts and why organisations might want to collaborate. However, it is the combination of local, national and global collaborations in CMI that provide organisations with long-lasting, futurethinking, well-rounded insights and deliverables.

Over the past few years, Saskatchewan, a Canada-based research and technology organisation's intelligence team has been making efforts to expand their intelligence network through collaborations. Based on these experiences, the authors provide an overview of the types of organisations that have been approached and the reasons behind each opportunity.

\section{Small and medium-sized businesses as well as large enterprises}

These organisations both within and outside of the key operating sectors provide access to 'on the ground' intelligence and are able to share insights on markets based on their intrinsic and vast experiences. Collaborations with key analysts, decision makers and other individuals offer the ability to understand various aspects of emerging local and national mega-trends relative to their sector, industry and area of focus.

\section{Economic development authorities and associations}

In discussion with representatives from these types of organisations, the shared intelligence is relative to local and national business drivers, attracting factors for new businesses and the retention of key businesses. These collaborations provide insights into economic indicators and trends mixed with industry-specific, sectoral and technological insights about what makes local businesses function and grow.

\section{Research agencies and institutes responsible for economic forecasting, public policy, skill development and organisational growth}

These collaborative efforts provide access to educational opportunities and thought leadership growth and expansion of the CI function locally and nationally. Through common workshops, training sessions, conference presentations and other such opportunities, organisations gain invaluable opportunities to grow one another's capabilities. Additionally, linkages with their expertise in economic forecasting, business growth and policy analysis add to the repertoire of sources and references.

\section{Educational institutions}

Connections with post-secondary institutions such as universities provide for proactive discussions revolving around growth and development of CMI capabilities within MBA, MSc, MLIS and other professional programmes. Collaborations in this sphere build an understanding of CI as it relates to business, research, STEM fields (Science, Technology, Engineering and Mathematics), R\&D and strategic decision making. By utilising CMI expertise and the university's ability to deliver programmes and training courses, today's new and emerging leaders gain access to information that was not previously available to them, or would not have been presented in such format and context. Several universities also work with CMI professional services companies and commercial organisations to provide surge research, project back-up and on-the-job training for their students.

\section{Professional service firms}

There are quite a few consulting organisations responsible for providing intelligence services to those who need them. Depending on each consultant, creative collaborative relationships can be established where one is not only paying for a service but also growing a long-term capacity and gaining access to resources, databases, expertise, thought leadership and networks that are non-existent in house. These collaborations help with challenging the norms of CMI practices, they push the way things are done and expand approaches to address complex problems. These organisations also grow networks as well as help the organisation to learn about, build and grow the intelligence function.

\section{Professional associations}

Linkages with professional associations that are responsible for driving intelligence forward as a profession or incorporate CMI into their training and growth, provide individuals with a readily available source of networks and thought leaders who have been in the field for a number of years. Through these collaborative networks, CMI practitioners learn and grow their function and capabilities, are challenged on their approaches and learn some of the real-world applications of intelligence. These networks provide many globally connected, long-lasting relationships.

\section{Other 'like' organisations}

Collaborative intelligence efforts have been established to advance the knowledge and reach of CI outside of the organisation as well as expand capabilities that other organisations might not have in-house. Once again, thought leaders and practitioners from these organisations provide their own perspectives of market intelligence and on decision making and together the efforts assist with the development and rise of new business models, as well as foresight and risk warning efforts that are objective, neutral and offer a 360-degree view of the operating environment. 


\section{Summary of inter-organisational collaboration}

The development of long-lasting collaborative intelligence efforts typically takes much time. It also takes a lot of effort, prior research and creative thinking to understand how the collaborations might work and what value they would add to the organisations at hand. Although time-consuming and work intensive, these collaborations can provide new perspectives, new ways of thinking and build capacity where sometimes none or little existed. Based on the authors' experiences, some of the top reasons why inter-organisational intelligence collaborations make sense and what they offer are illustrated in Table 1.

\section{Closing thoughts on inter-organisational collaboration}

Where could collaborations lead and what type of networks might be established next? Based on the need for intelligence from many stakeholders, the authors envision the possible creation of newly established industry-specific intelligence networks. These initiatives, likely taking the form of accelerators, catalysts, incubators or skunkworks, would consist of CI professionals, economists, service providers, technology developers, academia, scientists and engineers, strategists, business developers, legal, industry associations, government and others. Objectives of these networks would be to develop and grow key strategic sectors that are of importance to specific regions. These networks would focus not only on business and market intelligence in ensuring that businesses prosper and flourish but also would work towards creating environments where we would all be doing business in a setting that have social, environmental and economic impacts on communities locally, nationally and globally.

\section{A vision of the future of a collaborative CMI}

The authors' view of the future of CMI collaboration is promising. We see the following trends as being drivers over the next 5 years:

A shift will be towards collaborative CMI driving discussion and planning at community, state/province or nation-state/ country levels. The utilisation of findings and analytics will help drive policy development, support the growth of new funding and programming initiatives that will sustain and support and grow RD\&D, Innovation, Impacts and Social Responsibility.

The creation of new and effective Global Panels, Advisory Boards, Committees focusing on change, growth, innovation and overall positive impacts, will utilise insights generated by collaborative intelligence efforts through advising with 'topic leads' or by directly bringing collaborative CMI networks into their discussions and planning exercises.

Effective collaborative intelligence infrastructure and mobileenabled platforms will expand for soliciting ideas, generating discussions, sharing insights and developing new methodologies and foresight. Through this platform, boundaries that existed

TABLE 1: The advantages of inter-organisational collaborations.

Reason for collaborating
innovation,
Access to networks
Thought leadership
Breaking boundaries and historical
work patterns
Collaborative analytics
Bring together action-driven 'doers'
and foresight-driven 'strategists'
Pre-emptiveness
Develop a more 'all-inclusive' growth
environment

What collaboration offer?

Each organisation provides their expertise and viewpoint, challenging some of the norms and asking different types of questions. This can lead to new technological developments, changes to the way things are done within the organisation and develop new services. It is what each organisation takes away from the collaboration effort that is applicable and becomes invaluable.

Through the process of sharing of intelligence, organisations find that they are now also sharing their networks as those become relevant and applicable. Collaborations offer access to previously unavailable niche networks and information.

Organisations establish themselves as thought leaders relative to their areas of expertise and grow outside of a siloed environment. Gaining first- or second-hand access to thought leadership, new and developing knowledge and demonstrated practices allow for roadmaps permitting managed changes to occur that would otherwise be unknown.

Thought leaders in policy, economics and finance, technology development, legal and patents, providing their perspective on similar $\mathrm{KIQs}$, helps an organisation's CMI professionals develop 'complete' insights.

There are individuals in organisations who do not call themselves CMI professionals and might not have CMI in their job description, but they are doing CMI. There are also those who are constantly think up new ideas, new scenarios, see the bigger picture and are able to make assumptions on things that might happen in the future. These collaborations bring various individuals together who learn and elaborate on bringing the tactical and strategic together in realising some 'blue sky' ideas and implementing them into practice.

The next stage to being proactive is being pre-emptive - that is, building the future's organisations through which to do business in. These futures are rarely created by one organisation alone and through inter-organisational CMI collaboration, practitioners develop an understanding together of what the reality of the future might look like and what it will take from each of the many stakeholders to contribute to building that future into a reality.

environment

When building out a vision of the future and various scenarios that might play out, consideration is given to all the future stakeholders that might be a part of an event, even before it takes place. When assumed futures become realities and various stakeholders meet they now start with a similar and/or common mindset. Through the initial collaborations, 'right people get on the bus into their appropriate seats' much quicker (Collins 2001).

Develop a community of practice Through initial discussions, meetings and ideation stages, individuals involved in the collaborative networks develop a Community of Practice, where ideas and thoughts are shared, and processes are written and refined. These COPs provide a reference for other stakeholders to utilise and reference.

Full access to $\mathrm{Cl}$, while no or little $\mathrm{Cl}$ in-house

Organisations that become involved in these collaborative discussions might not have the need for or have the ability to have an in-house $\mathrm{CMI}$ individual or a team. Through collaboration these organisations are now able to gain access to a much broader community, much broader base of experts and thought leaders that provide insights and perspectives one would not have had access to otherwise.

Foresight and scenario-building capabilities

One of the main goals of $\mathrm{Cl}$ practitioners is to provide its clients with balanced and accurate insights to better enable their decision making. CMI aims to minimise risks and maximise the opportunities. Collaborators in CMI become better in thinking and planning forward. Executives or decision makers will not refuse insights that somewhat reliably help position their organisation as a leader in the near future.

Collaborations lead to working

Through collaborative $\mathrm{CMI}$, organisations create collaborative working relationships that lead to partnerships, as they explore and learn partnerships about one another's capabilities. CMI teams provide perspectives on how organisations can benefit from working together, establishing partnerships or collaborative relationships to deliver on a challenge based on their specific capabilities. 
in collaborating for intelligence will be pierced and individuals will be able to connect and tap into a vast array of professionals whose goals are to think and act strategically.

\section{Acknowledgements Competing interests}

The authors declare that they have no financial or personal relationships which may have inappropriately influenced them in writing this article.

\section{Authors' contribution}

C.F. and R.H. co-developed the conceptualisation, model building, inclusion of examples and samples, and execution of the article.

\section{References}

Choi, B.C. \& Pak, A.W., 2006, 'Multidisciplinarity, interdisciplinarity and transdisciplinarity in health research, services, education and policy: 1 . Definitions, objectives, and evidence of effectiveness', Clinical and Investigative Medicine 29(6), 351-364.

Collins, J.C., 2001, Good to great: Why some companies make the leap... and others don't, Random House, New York.

Cotton, P., 2012, What is good work? viewed 12 October 2015, from http://www. thinkers.sa.gov.au/lib/pdf/MartinSeligman/sowppts/BallC_1210_Cotton.pdf

Cotton, P. \& Hart, P., 2011, Positive psychology in the workplace, viewed 28 October 2015, from http://www.psychology.org.au/publications/inpsych/2011/april/ cotton/

Davenport, T.H., Harris, J.G. \& Morison, R., 2010, Analytics at work: Smarter decisions, better results, Harvard Business Press, Boston.

Fleisher, C.S., 2004, 'Competitive intelligence education: Competencies, sources, and trends', Information Management 38(2), 56-63.

Fleisher, C.S. \& Bensoussan, B.E., 2003, Strategic and competitive analysis: Methods and techniques for analyzing business competition, p. 457, Prentice Hall, Upper Saddle River, NJ.
Fleisher, C.S. \& Bensoussan, B.E., 2015, Business and competitive analysis: Effective application of new and classic methods, p. 590, FT Press, Upper Saddle River, NJ.

Fleisher, C.S., Wright, S. \& Allard, H.T., 2008, 'The role of insight teams in integrating diverse marketing information management techniques', European Journal of Marketing 42(7/8), 836-851. http://dx.doi.org/10.1108/03090560810877187

Gilad, B., 1994, Business blindspots: Replacing your company's entrenched and outdated myths, beliefs and assumptions with the realities of today's markets, Probus Professional Publishers, Chicago.

Hackman, J.R., 2002, Leading teams: Setting the stage for great performances, Harvard Business Press, Boston.

Hackman, J.R., 2011, Collaborative intelligence: Using teams to solve hard problems, Berrett-Koehler Publishers, San Francisco.

Hart, P.M. \& Cotton, P., 2003, 'Conventional wisdom is often misleading: Police stress within an organisational health framework', in M.F. Dollard, A.H. Winefield \& H.R. Winefeld (Eds.), Occupational Stress in the Service Professions, pp. 103-141, Taylor \& Francis, London.

Herring, J.P., 1999, 'Key intelligence topics: A process to identify and define intelligence needs', Competitive Intelligence Review 10(2), 4-14. http://dx.doi.org/10.1002/ (SICI)1520-6386(199932)10:2<4::AID-CIR3>3.0.CO;2-C

Heuer R.J., Jr., 2007, Small group processes for intelligence analysis. Unpublished manuscript, Sherman Kent School of Intelligence Analysis, Central Intelligence Agency. viewed from http://www.pherson.org/LibraryH,11

McDonald, J.P., Mohr, N., Dichter, A. \& McDonald, E.C., 2013, The power of protocols: An educator's guide to better practice, Teachers College Press, New York.

Prescott, J. \& Fleisher, C., 1991, 'SCIP: Who we are, what we do', Competitive Intelligence Review 2(1), 22-26. http://dx.doi.org/10.1002/cir.3880020112

Rao, T.V., 2014, HRD audit: Evaluating the human resource function for business improvement, Sage Publications, New Delhi, India.

Ross, C.S., 2003, 'The reference interview: Why it needs to be used in every (well, almost every) reference transaction', Reference \& User Services Quarterly 43(1), 38-43.

Rowley, J. \& Gibbs, P., 2008, 'From learning organization to practically wise organization', The learning organization 15(5), 356-372. http://dx.doi. org/10.1108/09696470810898357

Wagner, R. \& Harter, J.K., 2006, 12: The elements of great managing, Simon and Schuster, New York, Vol. 978, No. 1-59992.

West, C., 2001, Competitive intelligence, Palgrave Macmillan, London, UK.

Wilderom, C.P., Härtel, C.E., Ashkanasy, N.M., Vacharkulksemsuk, T., Sekerka, L.E., \& Fredrickson, B.L., 2011, 'Towards positive work cultures and climates', The Handbook of Organizational Culture and Climate 2, 79-84. http://dx.doi. org/10.4135/9781483307961.n5

Wood, D.J. \& Gray, B., 1991, 'Toward a comprehensive theory of collaboration', The Journal of Applied Behavioral Science 27(2), 139-162. http://dx.doi. org/10.1177/0021886391272001 